\title{
Ministry of Health Clinical Practice Guidelines: Diabetes Mellitus
}

\author{
Su Yen Goh, Seng Bin Ang, Yong Mong Bee, Richard YT Chen, Daphne Gardner, Emily Ho, Kala Adaikan, Alvin Lee, \\ Chung Horn Lee, Fong Seng Lim, Hwee Boon Lim, Su Chi Lim, Julie Seow, Abel Wah Ek Soh, Chee Fang Sum, \\ E Shyong $\underline{\text { Tai, }}$ Ah Chuan $\underline{\text { Thai, }}$ Tien Yin Wong, Fabian Yap
}

ABSTRACT The Ministry of Health $(\mathrm{MOH})$ have updated the clinical practice guidelines on Diabetes Mellitus to provide doctors and patients in Singapore with evidence-based treatment for diabetes mellitus. This article reproduces the introduction and executive summary (with recommendations from the guidelines) from the MOH clinical practice guidelines on Diabetes Mellitus, for the information of SMJ readers. Chapters and page numbers mentioned in the reproduced extract refer to the full text of the guidelines, which are available from the Ministry of Health website: http://www.moh. gov.sg/content/moh_web/healthprofessionalsportal/doctors/guidelines/cpg_medical.html. The recommendations should be used with reference to the full text of the guidelines. Following this article are multiple choice questions based on the full text of the guidelines.

\subsection{Objectives and scope of guideline}

The first edition of the $\mathrm{MOH}$ clinical practice guidelines on diabetes mellitus for Singapore was published in 1999. Since that time, more facts about this important condition have emerged, not only with regard to its diagnosis and treatment, but also about whether or not type 2 diabetes may be prevented, and, if so, how this may be achieved.

As diabetes mellitus has great public health significance in developed countries and developing nations alike, managing it properly involves a consideration, not just of clinical issues, but also of health economics. This second edition of the guidelines attempts to address some of these complex issues wherever evidence-based information pertaining to them is available.

\subsection{Target group}

The main aim of these guidelines is to help physicians make sound clinical decisions about diabetes mellitus by presenting up-to-date information about diagnosis, classification, treatment, outcomes, and follow-up.

These guidelines are developed for all health care professionals in Singapore. We hope they would be helpful especially to primary care physicians who care for patients with diabetes mellitus.

\subsection{Guideline development}

These guidelines have been produced by a committee of endocrinologists, family practitioners and primary care specialists, ophthalmologist, dietitian, social worker, and patient representative, appointed by the Ministry of Health. They were developed by the adaptation of existing guidelines, by the review of relevant literature and by expert clinical consensus with consideration of local practice. The guidance does not override the individual responsibility of healthcare professionals to make decisions appropriate to the circumstances of the individual patient, in consultation with the patient and/or guardian or carer.

\subsection{What's new in the revised guidelines}

The following is a list of the major revisions and additions to the previous guidelines:

- In Chapter 3, we have explained the rationale for criteria in diagnosing diabetes. In particular, for asymptomatic patients with a first test that meets criteria, we have attempted to provide more clarity on how to choose a second test, and how to interpret the findings.

- Chapter 4 is a new chapter which brings emphasis to two areas contributing towards positive outcomes in diabetes care: diabetes self-management education, and psychosocial assessment and holistic care of the person with diabetes.

- Chapter 5 on pharmacotherapy in diabetes mellitus has been updated to take into account recent clinical trial evidence of the efficacy of the newer classes of pharmacological agents.

- Chapter 6 focuses on glycaemic control, and emphasizes the importance of individualised targets, balancing the benefits of achieving targets without incurring undue risk of hypoglycaemia or other adverse effects, and considering the risk profile of the patient.

- In Chapter 7 on prevention of cardiovascular disease in diabetes mellitus, recommendations on decision-making in the area of therapeutics have been updated and harmonised with current local guidance on lipid, blood pressure and cardiovascular management. Target blood pressure 
ranges and LDL levels are discussed, as well as the role of antiplatelet therapy.

- Chapter 8 on prevention and management of diabetic nephropathy has been revised to present recent clinical trial evidence regarding the efficacy of, and indications for, the use of angiotensin-converting enzyme inhibitors and angiotensin receptor blockers.

- Chapter 9 on the prevention and management of eye complications has been updated to include developments such as intravitreal injection of anti-vascular endothelial growth factor in patients with diabetic macular oedema.

- Chapter 11 on pre-gestational and gestational diabetes has been updated. Women at high risk for gestational diabetes, but who are not found to have glucose intolerance in early pregnancy, are now recommended to be re-evaluated with a 75 gram OGTT at 24-28 weeks gestation.

- Chapter 13 is a new chapter outlining key principles in the management of the adult with type 1 diabetes, relevant to the primary care healthcare professional.

\subsection{Review of guidelines}

Evidence-based clinical practice guidelines are only as current as the evidence that supports them. Users must keep in mind that new evidence could supersede recommendations in these guidelines. The workgroup advises that these guidelines be scheduled for review four years after publication, or earlier if new evidence emerges that necessitates substantive changes to the recommendations.

Future revisions may include management of hypoglycaemia in persons with diabetes, and evolving areas like bariatric surgery and pancreas/islet cell transplantation.

\section{EXECUTIVE SUMMARY OF RECOMMENDATIONS}

Details of the recommendations listed can be found in the main text as the pages indicated.

\section{Diagnosis and screening of diabetes mellitus in Singapore}

D In patients with hyperglycaemic crisis, diabetes mellitus can be diagnosed without further testing (pg 42).

\section{Grade D, Level 4}

B In patients with typical symptoms, diabetes mellitus can be diagnosed if any one of the following is present.

1. Casual plasma glucose $>11.1 \mathrm{mmol} / \mathrm{l}$

2. Fasting plasma glucose $>7.0 \mathrm{mmol} / \mathrm{l}$

3. 2-hour post-challenge plasma glucose $>11.1 \mathrm{mmol} / \mathrm{l}$

Other individuals should have a repeat test on a subsequent day (pg 42).

Grade B, Level 2++

When two different tests are available for the same patient and the results for both tests are above the diagnostic thresholds, the diagnosis of diabetes is confirmed (pg 42).
D When two different tests are available in an individual and the results are discordant, the test whose result is above the diagnostic cut point (usually the fasting plasma glucose or 2-hour post-challenge glucose) should be repeated (pg 42).

Grade D, Level 4

D Fasting plasma glucose measured in an accredited laboratory is the preferred test for the diagnosis of diabetes mellitus (pg 43).

Grade D, Level 4

B All subjects with fasting plasma glucose from 6.1 to $6.9 \mathrm{mmol} / \mathrm{l}$ should undergo a $75 \mathrm{~g}$ oral glucose tolerance test to determine if they have impaired glucose tolerance or diabetes mellitus (pg 43).

Grade B, Level 2++

D If a second test fails to confirm the diagnosis, barring a laboratory error, such patients are likely to have test results near the margins of the threshold for a diagnosis. The healthcare professional might opt to follow the patient closely and repeat the testing in 6-12 months (pg 43).

Grade D, Level 4

$\mathrm{HbA}_{1 \mathrm{c}}$ is not recommended as a screening and diagnostic tool for diabetes mellitus until its performance in our multiethnic population has been evaluated (pg 43)

GPP

B Intermediate states of glucose metabolism termed impaired fasting glucose and impaired glucose tolerance should be recognised as defined in Table 1 (pg 46).

Grade B, Level $2^{++}$

D Screening should be considered in adults of any age who have one or more risk factors for diabetes. In those without risk factors, testing should begin at 40 years (pg 47).

Grade D, Level 4

D Subsequently, screening should be carried out every three years for those with normal glucose tolerance and annually for those with impaired fasting glycaemia (IFG) or impaired glucose tolerance (IGT) (pg 47).

Grade D, Level 4

\section{Lifestyle modification}

D Individuals who have diabetes should receive individualised medical nutritional therapy as needed to achieve treatment goals, preferably provided by a dietitian familiar with the components of diabetes medical nutrition therapy (pg 49).

Grade D, Level 4

Special attention should be paid to the diabetic patient's dietary requirements during periods of sickness, fasting, travel and exercise (pg 49). 
D A diet for diabetes should contain a good balance of carbohydrate, protein and fat, adjusted to meet the individual's metabolic goals and preferences (pg 50).

Grade D, Level 4

D Individualised meal planning for diabetes should include optimisation of food choices to meet recommended dietary allowance for all micronutrients, providing adequate vitamins and minerals (pg 50).

Grade D, Level 4

B Meal and snack carbohydrate intake for diabetes should be consistently distributed throughout the day, on a day to day basis, as consistency in carbohydrate intake has been shown to result in improved glycaemic control (pg 50).

Grade B, Level $2^{+}$

D Consumption of macronutrients is based on recommended dietary allowance (RDA) for healthy eating; $50-60 \%$ of total energy from carbohydrates should be encouraged (pg 50).

Grade D, Level 4

B If weight reduction is needed, it should be attempted gradually (0.25 to $1.0 \mathrm{~kg} /$ week). In overweight or obese patients with type 2 diabetes, a weight loss of $5-10 \%$ of body weight achieved through lifestyle interventions is a realistic goal (pg 51).

Grade B, Level 2+

D Dietary protein intake of approximately $15-20 \%$ of daily energy intake is appropriate for most patients with type 2 diabetes (pg 51).

Grade D, Level 4

D It is recommended that total calories from fat intake be kept to $<30 \%$ of total calorie intake in diabetic patients (pg 51).

Grade D, Level 4

D Trans fats should be limited to $1 \%$ of total energy intake and cholesterol intake ( $<200 \mathrm{mg}$ daily) to reduce risk for cardiovascular disease. These goals are similar for individuals with pre-existing cardiovascular disease (pg 52).

Grade D, Level 4

B Recommendations for fibre intake for people with diabetes are similar to the recommendation for the general population. A daily consumption of a diet containing 20-35 g of dietary fibre from a wide variety of food sources is recommended (pg 52).

Grade B, Level $2^{+}$

D Sodium intake should be restricted to $<2 \mathrm{~g}$ per day for diabetic individuals with hypertension (pg 52).

Grade D, Level 4

D Diabetes patients with poor glycaemic control or are overweight should abstain from alcohol. If individuals choose to drink, intake should be limited to a moderate amount, as per the general population (no more than two drinks for women per day and no more than three drinks per day for men) (pg 52).

Grade D, Level 4

B

Individuals who choose to use non-nutritive sweeteners should be advised that some of these products might contain energy and carbohydrate from sources that might need to be accounted for (pg 52).

\section{Grade B, Level $2^{+}$}

For exercise more vigorous than brisk walking, a preexercise physician evaluation is recommended for individuals with diabetes to identify cardiovascular risks and any complications of severe neuropathy or severe diabetic retinopathy that may contraindicate certain activities and predispose to injury (pg 53).

Grade D, Level 4

Individuals with severe proliferative diabetic retinopathy should avoid activities that greatly increase intraocular pressure and risk of haemorrhage (pg 54).

Grade D, Level 3

Individuals with peripheral neuropathy and without acute ulceration may participate in moderate weight-bearing exercise. Comprehensive foot care, use of appropriate footwear and daily foot check is recommended (pg 54).

Grade B, Level 2+

Individuals with type 2 diabetes should undertake at least 150 mins/week of moderate to vigorous aerobic exercise spread out during at least 3 days of the week, with no more than 2 consecutive days between bouts of exercise (pg 54).

Grade B, Level $1^{+}$

Individuals with diabetes, especially those on insulin treatment or secretagogues, may require medication dose adjustments and should receive specific education on the prevention of exercise induced hypoglycaemia (pg 55).

Grade D, Level 4

Individuals with diabetes should be encouraged to stop smoking (pg 55).

Grade C, Level 3

People with diabetes should receive Diabetes SelfManagement Education (DSME) when their diabetes is diagnosed and as needed thereafter (pg 57).

Grade B, Level $2^{+}$

Assessment of psychological and social wellbeing should be included as an ongoing part of diabetes management (pg 59). 
D Clinicians should provide the following psychosocial support to patients during the diagnosis phase of diabetes management:

- Provide medical information and psychological support.

- Be accessible and sensitive to patient's needs.

- Provide information and repeat if necessary as they may not retain much at this stage.

- Introduce to other patients to get them support and an accepting environment

- Involve other family members if necessary (pg 59).

Grade D, Level 4

D Clinicians should provide the following psychosocial support to patients during the maintenance phase of diabetes management:

- Motivate patient and family to maintain optimal control

- Create an individualised workable regimen and help patient adhere to it

- Ensure good support from diabetes team

- Check for signs of diabetes burnout

- Consider educational intervention

- Follow up and review behavioural changes

- Modify treatment if necessary (pg 60).

Grade D, Level 4

D Clinicians should provide the following psychosocial support to patients during the complications phase of diabetes management:

- Giving them the space to vent and providing them with a lot of realistic reassurance is important

- Do not overwhelm with information but allow for grieving first

- Gentle motivation to encourage patients to maintain adherence to treatment regimen and possibly revising some of the information or education will be helpful

- Counselling is important but needs to be timely (pg 60).

Grade D, Level 4

D Patients with diabetes should be encouraged to find support from other persons and families living with diabetes and community programmes which reinforces diabetes education and promotes living well with diabetes. These community based programmes provide a safe and accepting environment for learning and sharing with others who live with the same condition (pg 60).

Grade D, Level 4

\section{Pharmacotherapy}

B Long-acting sulphonylureas e.g., chlorpropamide and glibenclamide, carry a high risk of hypoglycaemia and are not recommended (pg 62).

Patients with type 2 diabetes may initially be treated with lifestyle modification (diet and exercise) unless they are symptomatic or severely hyperglycaemic (i.e. random blood glucose $>15 \mathrm{mmol} / \mathrm{l}$ or fasting blood glucose $>10 \mathrm{mmol} / \mathrm{l}$ ) in which case pharmacological therapy should be initiated together with lifestyle intervention (pg 63).

Grade A, Level $1^{+}$

Oral glucose lowering agents should be started if glycaemic targets are not achieved in a timely and appropriate manner (pg 63).

Grade A, Level $1^{+}$

If glycaemic targets are not reached with a single oral agent, combination therapy with one or more agents (including insulin) from other classes may be considered. However, one would need to monitor carefully for adverse events such as hypoglycaemia or fluid retention (pg 63).

Grade A, Level $1^{+}$

Insulin therapy should be considered, if optimal combination therapy fails to attain target control (i.e., 2 consecutive $\mathrm{HbA}_{1 \mathrm{c}}$ values failed to reach $\leq 8 \%$ over 3-6 months interval) (pg 63).

Grade A, Level 1+

$A$

Metformin is usually considered first-line pharmacotherapy, and sulphonylureas/dipeptidyl peptidase 4 (DPP-IV) inhibitors/alpha-glucosidase inhibitors are reasonable alternatives as first-line pharmacotherapy (pg 64).

Grade A, Level $1^{+}$

For type 2 diabetes, two or more oral agents, or insulin therapy, either alone or in combination with oral agents, may be required (pg 64).

Grade A, Level 1+

For type 2 diabetes, other oral agents are acceptable alternatives to metformin as initial monotherapy, if the person does not tolerate metformin, or where metformin is contraindicated (pg 64).

Grade A, Level $1^{+}$

In the setting of severely uncontrolled type 2 diabetes (for example, $\mathrm{HbA}_{1 \mathrm{c}}>10 \%$, random glucose levels consistently above $16.7 \mathrm{mmol} / \mathrm{L}$ ), the presence of ketonuria, or symptomatic diabetes with polyuria, polydipsia and weight loss, insulin therapy in combination with lifestyle intervention may be the initial treatment of choice (pg 64).

Grade A, Level $1^{+}$ In elderly patients, initiating therapy with low-dose, shortacting oral glucose lowering agents is recommended (pg 65).

Grade D, Level 4 
D Metformin is usually contraindicated in the presence of severe renal or hepatic insufficiency as it may be associated with lactic acidosis (pg 65).

Grade D, Level 4

D It is advisable to use metformin with caution in those at risk of a sudden deterioration in renal function and those with eGFR $<45 \mathrm{ml} / \mathrm{min} / 1.73 \mathrm{~m}^{2}$ and to cease metformin usage if the eGFR is below $30 \mathrm{ml} / \mathrm{min} / 1.73 \mathrm{~m}^{2}$ (pg 65).

Grade D, Level 4

D Metformin must be used with care in the presence of comorbid conditions which increase the risk of lactic acidosis (e.g., class III or IV cardiac failure) (pg 66).

Grade D, Level 4

D Thiazolidinediones (in particular, rosiglitazone) are contraindicated in patients with acute coronary syndrome, ischaemic heart disease, and all classes of heart failure (including New York Heart Association (NYHA) Functional Classification Class I/II heart failure patients) and are also not recommended for use in patients with peripheral arterial disease (pg 66).

Grade D, Level 4

GPP Treatment choices should be individualised and culturally appropriate, and patients should have the opportunity to make informed decisions on their care and treatment options, in partnership with their healthcare providers (pg 67).

GPP

D The use of exenatide is not recommended in type 2 diabetes patients with a history of pancreatitis (pg 68).

Grade D, Level 4

A All patients with type 1 diabetes must receive insulin. Multiple daily injections (3 or more) or the use of continuous subcutaneous insulin infusion (CSII or insulin pump therapy) may be required to achieve target glucose levels (pg 68).

Grade A, Level $1^{+}$

D Patients on insulin must be equipped not only with the skills of insulin administration, but also should be educated on self-monitoring of blood glucose, hypoglycaemia management, matching of insulin dose and carbohydrate intake, and dose adjustments during sick days, travel, exercise, and changes in food intake (pg 68).

Grade D, Level 4

B Insulin therapy should be managed with relevant and regular insulin and hypoglycaemia-related selfmanagement training with the common goal of improved glycaemic control and reduction in risk of severe hypoglycaemia (pg 69).

Grade B, Level $2^{++}$
D In type 2 diabetes, introduction of insulin should not be delayed if metabolic control becomes suboptimal. This may be initiated as a bedtime dose of intermediate-acting or long-acting insulin with maintenance of oral agents during the day (pg 75).

Grade D, Level 4

When glycaemic control is not achieved despite the addition of basal insulin to oral agents, discontinuing sulphonylureas and switching to premixed twice daily or basal-bolus insulin regimens becomes necessary. However, metformin and $\alpha$-glucosidase inhibitors may still be used in conjunction with exogenous insulin to attenuate the insulin dose. Fine-tuning of insulin doses is best determined by home blood glucose monitoring. Patients with type 2 diabetes who are switched to insulin therapy temporarily during episodes of acute stress, such as sepsis, may be put back on oral agents when their glycaemic control improves with declining insulin resistance and glucotoxicity (pg 75).

Grade D, Level 4

\section{Glycaemic control: assessment and targets}

B Self-monitoring of blood glucose is recommended for patients with type 1 or type 2 diabetes who are using insulin (pg 77).

Grade B, Level $2^{++}$

D Self-monitoring of blood glucose should be considered in the following groups of patients with type 2 diabetes who are not treated with insulin:

- Those at increased risk of developing hypoglycaemia or its consequences (e.g., patients who are using sulphonylureas)

- Those pregnant patients with pre-existing diabetes or gestational diabetes

- Those experiencing acute illness

- Those who have failed to achieve glycaemic goals

- Those undergoing fasting, for example, during Ramadan (pg 78).

Grade D, Level 4

B Self-monitoring of blood glucose should be carried out 3 or more times daily for patients with type 1 diabetes (pg 79).

Grade B, Level $2^{++}$

GPP For patients with unstable metabolic control, changes in daily routine, alterations of treatment regimens or acute illness, the frequency of self-monitoring of blood glucose should be increased (pg 79).

GPP

Health care professionals should be familiar with the practical use of glucometers (pg 79).

GPP 
B To ensure optimal benefit from self-monitoring of blood glucose, patients must be educated on the interpretation of glucose levels (pg 80).

Grade B, Level 1+

Periodic reviews are recommended to verify users' competency, together with comparisons between results from patient self-testing of blood glucose in the clinic and simultaneous laboratory testing (pg 80).

GPP It is recommended that calibration checks of meters are periodically conducted using standard solutions according to the manufacturer's recommendations (pg 80).

GPP

D Continuous glucose monitoring (CGM) may be used as a supplemental tool to SMBG in patients with hypoglycaemia unawareness and/or frequent hypoglycaemic episodes (pg 81).

Grade D, Level 3

B Self-monitoring of urine glucose is not recommended for monitoring of glycaemic status (pg 81).

Grade B, Level $1^{+}$

A Ketone monitoring should be performed during sustained hyperglycaemia (e.g., blood glucose > $14.0 \mathrm{mmol} / \mathrm{l}$ ) in patients with type 1 diabetes, especially during acute illness. Blood ketone monitoring is preferable to urine ketone monitoring (pg 81).

Grade A, Level $1^{+}$

D Glycated haemoglobin $\left(\mathrm{HbA}_{1 c}\right)$ should be performed routinely in all patients with diabetes, at initial assessment and then as part of follow-up care (pg 82).

Grade D, Level 4

D The measurement of $\mathrm{HbA}_{1 \mathrm{c}}$ should be done in laboratories that utilise DCCT-aligned assays (DCCT - Diabetes Control and Complications Trial) (pg 83).

Grade D, Level 4

The following schedule is recommended for $\mathrm{HbA}_{1 \mathrm{c}}$ testing in patients with diabetes:

- 3- to 4-monthly in patients with unstable glycaemic control, failure to meet treatment goals, recent adjustment in therapy, or intensive insulin therapy.

- 6-monthly in patients who have stable glycaemic control and who are meeting treatment goals (pg 83).

Grade D, Level 4

B $\quad \mathrm{HbA}_{1 \mathrm{c}}$ result should be made available at the time that the patient with diabetes is seen (pg 83).

Grade B, Level $2^{++}$
D The targets of glycaemic control should be individualised (pg 83).

Grade D, Level 4

Patients should participate in the process of defining their targets of glycaemic control (See Table 6) (pg 84).

GPP

A The $\mathrm{HbA}_{1 \mathrm{c}}$ target for most non-pregnant adults with type 1 or type 2 diabetes should be $\leq 7.0 \%$ or $\leq 53 \mathrm{mmol} / \mathrm{mol}$ (pg 84).

Grade A, Level $1^{++}$

B Lowering $\mathrm{HbA}_{1 \mathrm{c}}$ target to $\leq 6.5 \%$ or $\leq 47.5 \mathrm{mmol} / \mathrm{mol}$ may be considered for some patients with type 2 diabetes at doctor and patient judgement, if this can be achieved without significant hypoglycaemia. Such patients include those with short duration of diabetes, long life expectancy and no significant cardiovascular disease (pg 85).

Grade B, Level 1+

D Less stringent $\mathrm{HbA}_{1 \mathrm{c}}$ target (e.g., 7.0 to $8.5 \%$ or $53 \mathrm{mmol} / \mathrm{mol}$ to $69.4 \mathrm{mmol} / \mathrm{mol}$ ) may be adopted for some patients vulnerable to the harmful effects associated with tight glycaemic control. Such patients include those with very long duration of diabetes, known history of severe hypoglycaemia, advanced atherosclerosis and advanced age (pg 85).

Grade D, Level 4

Doctors should be vigilant in preventing hypoglycaemia by reviewing treatment regimens in patients with near-normal $\mathrm{HbA}_{1 \mathrm{c}}$ levels (e.g., $<6.0 \%$ or $42.1 \mathrm{mmol} / \mathrm{mol}$ ), especially those treated with insulin or insulin secretagogues (pg 86).

GPP

\section{Prevention of cardiovascular disease in people with diabetes mellitus}

GPP The assessment of cardiovascular risk in persons with type 2 diabetes mellitus should include:

History - which should include:

- Smoking

- Hypertension

- Pre-existing cardiovascular disease (including angina, myocardial infarction, stroke, PAD)

- Family history of premature coronary artery disease (non-modifiable).

Physical examination - which should include:

- Assessment for peripheral vascular disease

- Measurement of blood pressure at every visit.

Tests - which should include:

- Fasting serum lipids at or soon after diagnosis and at least annually

- Urine microalbumin or protein at least annually

- Serum creatinine and estimation of eGFR (See chapter 8) 
- Electrocardiogram (resting) routinely at baseline. Subsequent ECG may be performed when clinically indicated (pg 88).

B For patients with type 2 diabetes mellitus who have hypertension, an acceptable treatment-initiation and target blood pressure is <140/80 mm Hg (pg 91).

\section{Grade B, Level $2^{+}$}

B An angiotensin-converting-enzyme (ACE) inhibitor or angiotensin receptor blocker (ARB) should be included as part of antihypertensive regimen for people with type 2 diabetes requiring pharmacotherapy for hypertension, unless not well tolerated (pg 93).

Grade B, Level 2+

D All persons with type 2 diabetes mellitus should have a full lipid profile, including low density lipoprotein (LDL) cholesterol, fasting triglyceride and high density lipoprotein (HDL) cholesterol, measured at the time of diagnosis. These should be obtained after 10-12 hours of fasting (pg 96).

Grade D, Level 4

D If optimal, serum lipids should be measured 12-monthly in persons with type 2 diabetes (pg 96).

Grade D, Level 4

D The majority of patients with type 2 diabetes mellitus should have a primary low density lipoprotein (LDL) cholesterol goal $<2.6 \mathrm{mmol} / \mathrm{L}$ and should receive medical nutrition and pharmacological therapy to achieve this goal (pg 96).

Grade D, Level 4

D Patients with diabetes who have overt cardiovascular disease and/or chronic kidney disease but are not on maintenance hemodialysis should have low density lipoprotein (LDL) cholesterol lowered with combination of dietary and pharmacological means to a target of $<2.1$ $\mathrm{mmol} / \mathrm{L}$ (pg 96).

Grade D, Level 4

D When making a therapeutic decision with the patient, the potential benefits of adding/increasing lipid-lowering pharmacological treatment, needs to be considered together with the potential risks of such treatment (pg 96).

Grade D, Level 4

D For most patients with type 2 diabetes mellitus where low density lipoprotein ( $\mathrm{LDL}$ ) cholesterol is $>2.6 \mathrm{mmol} / \mathrm{L}$, an HMG CoA reductase inhibitor (statin) should be started concurrently with therapeutic lifestyle modification (pg 97).

Grade D, Level 4

D It is reasonable to initiate low dose aspirin for primary prevention in people with diabetes and no previous history of vascular disease at age 50 years for men, and 60 years for women, provided they also have at least one more of the following cardiovascular risk factors: smoking, hypertension, dyslipidaemia, family history of premature cardiovascular disease and albuminuria (pg 99).

Grade D, Level 4

In the presence of aspirin allergy, other antiplatelet agents such as clopidogrel (75 mg per day) may be a reasonable alternative for patients with high risk (pg 99).

GPP

\section{Diabetic nephropathy - screening and treatment}

D It is recommended to perform an annual test to assess urine albumin excretion in type 1 diabetic patients with diabetes duration of 5 years and in all type 2 diabetic patients, starting at diagnosis (pg 101).

Grade D, Level 4

D Measure serum creatinine at least annually in all adults with diabetes (regardless of the degree of urine albumin excretion) is recommended. The serum creatinine should be used to estimate glomerular filtration rate (GFR) and stage the level of chronic kidney disease (CKD), if present (pg 102).

Grade D, Level 4

It is only recommended to estimate renal function with the Modification of Diet in Renal Disease (MDRD) equation when eGFR is below $60 \mathrm{mls} / \mathrm{min} / 1.73 \mathrm{~m}^{2}$ (pg 103).

Grade C, Level $2^{+}$

To reduce the risk or slow the progression of nephropathy, optimised glucose control is recommended (pg 104).

Grade A, Level 1+

To reduce the risk or slow the progression of nephropathy, optimised blood pressure control is recommended (pg 104).

Grade A, Level $1^{+}$

A It is recommended that in the treatment of the nonpregnant patient with micro- or macroalbuminuria, either angiotensin-converting enzyme (ACE) inhibitors or angiotensin receptor blockers (ARBs) should be used (pg 105).

Grade A, Level $1^{+}$

In patients with type 1 diabetes, with hypertension and any degree of albuminuria, angiotensin-converting enzyme (ACE) inhibitors are recommended (pg 105).

Grade A, Level $1^{+}$

A In patients with type 2 diabetes, hypertension, and microalbuminuria, angiotensin-converting enzyme (ACE) 
inhibitors or angiotensin receptor blockers (ARBs) are recommended (pg 105).

Grade A, Level 1+

A In patients with type 2 diabetes, hypertension, macroalbuminuria, and renal insufficiency (serum creatinine $>1.5 \mathrm{mg} / \mathrm{dl}$ ), angiotensin receptor blockers (ARBs) are recommended (pg 105).

Grade A, Level $1^{+}$

D In patients with diabetes, if one class [either angiotensinconverting enzyme (ACE) inhibitors or angiotensin receptor blockers (ARBs)] is not tolerated, the other should be substituted (pg 105).

Grade D, Level 4

D When angiotensin-converting enzyme (ACE) inhibitors, angiotensin receptor blockers (ARBs) or diuretics are used, it is recommended to monitor serum creatinine and potassium levels for the development of acute kidney disease and hyperkalemia (pg 106).

Grade D, Level 4

A To reduce the risk or slow the progression of nephropathy, optimised lipid control is recommended (pg 106).

Grade A, Level 1+

A Reduction of protein intake to $0.8-1.0$ g per $\mathrm{kg}$ body wt per day in individuals with diabetes and earlier stages of chronic kidney disease (CKD) and to 0.8 g per kg body wt per day in the later stages of CKD is recommended to improve measures of renal function (urine albumin excretion rate, GFR) (pg 107).

Grade A, Level $1^{+}$

A It is recommended to consider a low-dose aspirin in diabetic individuals with a history of vascular disease (pg 107).

Grade A, Level $1^{+}$

D It is recommended to consider low-dose aspirin in diabetic individuals who carry significant cardiovascular risk burden (pg 107).

Grade D, Level 4

D Continual monitoring of urine albumin excretion to assess both response to therapy and progression of disease is recommended for both type 1 and type 2 diabetes patients (pg 108).

Grade D, Level 4

D It is recommended that when estimated GFR (eGFR) is $<60 \mathrm{ml} . \mathrm{min} / 1.73 \mathrm{~m}^{2}$, evaluate and manage potential complications of Chronic Kidney Disease (CKD) (pg 108).

Grade D, Level 4

It is recommended to consider referral to a physician experienced in the care of kidney disease when there is

uncertainty about the etiology of kidney disease (heavy proteinuria, active urine sediment, absence of retinopathy, rapid decline in GFR), difficult management issues, or advanced kidney disease (pg 108).

Grade D, Level 4

\section{Prevention and management of eye complications}

D All patients diagnosed with diabetes require regular visual acuity assessment and eye examinations by trained personnel to screen for diabetic retinopathy using a test of adequate sensitivity (pg 110).

Grade D, Level 4

D Retinal screening preferably using retinal photography or direct ophthalmoscopy (if retinal photography is not available) through dilated pupils is recommended (pg 110).

Grade D, Level 4

Type 1 diabetic patients should have an eye examination 3-5 years after diagnosis of diabetes, and at least once yearly subsequently. Type 2 diabetic patients should have an ocular assessment at the time of diagnosis and at least once yearly subsequently (pg 111).

Grade D, Level 4

Women with diabetes mellitus who intend to have children should preferably have an eye examination prior to conception, followed by one during the early first trimester. Further eye examinations during pregnancy may be done depending on the results of the first trimester examination (pg 111).

Grade D, Level 4

A or 47.5 to $58.5 \mathrm{mmol} / \mathrm{mol}$ ) should be instituted to reduce the risk and progression of diabetic retinopathy (pg 113).

Grade A, Level $1^{+}$

Rapid normalisation of blood glucose may worsen retinopathy and thus retinal assessment should be carried out before initiation of intensive insulin therapy and then at 3-monthly intervals for 6-12 months. Patients should be carefully monitored during this period (pg 114).

Grade D, Level 3

A Good control of blood pressure at or below 130/80 mm Hg should be instituted to reduce the risk and progression of diabetic retinopathy (pg 114).

Grade A, Level $1^{+}$

B Significant hyperlipidaemia should be treated to retard diabetic retinopathy. Consideration should be given to using fenofibrate (pg 115).

Grade B, Level $1^{+}$

Diabetic patients found to have diabetic macular oedema or moderate and more severe non-retinopathy by their 
physicians should be referred for further ophthalmological assessment (pg 117).

A Timely laser therapy should be offered to patients with proliferative diabetic retinopathy and diabetic macular oedema (pg 118).

\section{Grade A, Level 1+}

Diabetic patients with visual acuity of $6 / 18$ or worse or with diabetic macular oedema should be referred for further ophthalmological assessment (pg 118).

A Pan-retinal laser photocoagulation should be instituted for severe and proliferative diabetic retinopathy as it produces a $50 \%$ reduction in risk for severe visual loss and need for vitrectomy (pg 119).

Grade A, Level 1+

A Focal/grid laser photocoagulation should be instituted for diabetic macular oedema as it results in a $50 \%$ reduction in risk for moderate visual loss (pg 119).

Grade A, Level $1^{+}$

A Intravitreal injection of anti-vascular endothelial growth factor (anti-VEGF) agents may be offered to patients with diabetic macular oedema, particularly in cases where laser photocoagulation has not been effective (pg 120).

Grade A, Level $1^{+}$

B Vitrectomy may be offered to selected patients with advanced diabetic retinopathy (pg 121).

Grade B, Level $1^{+}$

\section{Prevention of diabetic foot complications}

B All individuals with diabetes should receive an annual foot examination to identify high-risk foot conditions (pg 124).

Grade B, Level 2+

B The assessment of the feet involves risk identification, treatment and patient education appropriate to the level of risk (pg 124).

Grade B, Level 2+

GPP All patients, regardless of risk category, should receive ongoing education on footcare and footwear advice (pg 124).

C Patients identified with foot-related risk conditions should have access to a specialised footcare team which should include diabetes specialist, podiatrist, physiotherapist trained in diabetes, diabetes nurse educator, vascular and orthopaedic surgeons (pg 126).

Grade C, Level 3
Urgent referral to a specialised footcare team is needed in the presence of ulcerations, severe foot infection and gangrene (pg 128).

\section{Management of women with pre-gestational and gestational diabetes mellitus}

B All women with diabetes in the reproductive age group should receive prepregnancy counselling, particularly before starting a family (pg 132).

Grade B, Level 1'

Wherever possible, prepregnancy counselling should be performed jointly by a multi-disciplinary team skilled in diabetes care, including the physician, obstetrician, dietician, nurse-educator and other specialists (pg 133).

GPP

Risk assessment for gestational diabetes should be undertaken at the first antenatal visit (pg 133).

Grade D, Level 4

Women at high-risk for gestational diabetes (GDM) should undergo an oral glucose tolerance test (OGTT) as early in pregnancy as feasible. Re-evaluation should be performed at 24-28 weeks of gestation if glucose intolerance is not present at the early screen (pg 134).

Grade B, Level 1+

In pregnant women who are not at high risk for gestational diabetes, urine for glucose should be obtained at each antenatal visit and random blood sugar levels ascertained when there is $\geq 1+$ glycosuria. A diagnostic test is necessary if the random plasma blood glucose $>6.6 \mathrm{mmol} / \mathrm{l}$ more than 2 hours after a meal, or $>7.0 \mathrm{mmol} / \mathrm{l}$ within 2 hours of a meal (pg 135).

Grade D, Level 3

Gestational diabetes is diagnosed with a $75 \mathrm{~g}$ oral glucose tolerance test (OGTT). A fasting venous plasma glucose $\geq 7.0 \mathrm{mmol} / \mathrm{l}$ or a 2 -hour venous plasma glucose of $\geq 7.8$ $\mathrm{mmol} / \mathrm{l}$ is diagnostic of gestational diabetes. Casual venous plasma levels $\geq 11.1 \mathrm{mmol} / \mathrm{l}$ on 2 successive occasions would confirm gestational diabetes without recourse to oral glucose tolerance testing (pg 135).

Grade B, Level 1
All women diagnosed with pregestational diabetes and those diagnosed with gestational diabetes should receive specialised care (pg 135).

Grade D, Level 3 the first instance to attain glycaemic goals. Sweet foods should be avoided and caloric intake reduced if the woman is overweight or obese. The diet should contain more 
complex carbohydrates, more fibre, and less saturated fat. Nutritional counselling should be individualised, taking into account the patient's body weight, weight gain and physical activity (pg 136).

Grade B, Level $2^{++}$

B If nutritional therapy does not consistently maintain a fasting or pre-meal capillary blood glucose of $<5.5 \mathrm{mmol} / /$ and/or a 1-hour postprandial capillary glucose of $<7.8$ $\mathrm{mmol} / /$ or a 2-hour postprandial capillary blood glucose of $<6.7 \mathrm{mmol} / \mathrm{l}$ on two or more occasions within a 1-2 week interval, insulin therapy should be considered (pg 136).

Grade B, Level $2^{++}$

C In pregestational diabetes, individualised intensive (multidose) insulin therapy is often necessary to achieve and maintain target blood glucose levels (pg 137).

Grade C, Level 2+

D Maintain maternal capillary blood glucose concentrations as near normal as possible at $<5.5 \mathrm{mmol} / \mathrm{l}$ in the fasting or pre-meal state, and/or $<7.8 \mathrm{mmol} / \mathrm{l}$ one hour after meals, or $<6.7 \mathrm{mmol} / \mathrm{l}$ two hours after meals (pg 137).

Grade D, Level 4

D Self-monitoring of blood glucose (SMBG) is essential during pregnancy for women with gestational diabetes and pregestational diabetes. Both preprandial and postprandial testing are recommended to guide therapy in order to achieve glycaemic targets (pg 137).

Grade D, Level 3

D Women with pregestational type 1 diabetes should be advised to test for ketonuria or ketonaemia if they become hyperglycaemic or unwell (pg 137).

Grade D, Level 4

D Oral glucose-lowering drugs are not recommended during pregnancy under normal circumstances. Women with pregestational type 2 diabetes who become pregnant while taking oral glucose-lowering drugs should be switched to insulin therapy (pg 138).

Grade D, Level 4

D An early pregnancy scan should be performed to confirm viability and accurately date the pregnancy in women with pregestational diabetes, especially when glycaemic control is suboptimal or changes in medications are required (pg 138).

Grade D, Level 4

B A detailed foetal anomaly scan, including four-chamber cardiac view and outflow tracts, should be performed between 18-22 weeks in women with pregestational diabetes or when overt diabetes is diagnosed in the early pregnancy (pg 138).
Women with pregestational diabetes and gestational diabetes should be offered ultrasound monitoring of foetal growth (foetal abdominal circumference and/or estimated foetal weight) and amniotic fluid volume every 4 weeks from 28 to 36 weeks (pg 139).

Grade D, Level 4

Mothers with gestational diabetes mellitus and pregestational diabetes should be taught to monitor foetal movements during the last 10-12 weeks of pregnancy and to report immediately any reduction in the perception of foetal movements (pg 139).

Grade D, Level 4

C

Non-stress testing with cardiotocography and umbilical doppler flow studies may be considered in cases where hyperglycaemia warrants insulin therapy and in cases where other high-risk factors are present (pg 139).

Grade C, Level $2^{+}$

C

In women with gestational diabetes mellitus as well as those with pregestational diabetes, the measurement of blood pressure and dipstick testing for urinary protein is recommended at each antenatal visit to detect the development of pregnancy-induced hypertension and preeclampsia, especially if there is pre-existing nephropathy (pg 139).

Grade C, Level $2^{+}$

Women with pregestational diabetes should have their serum creatinine and electrolytes assessed at the first antenatal visit and in the third trimester (pg 139).

GPP

For women with pregestational diabetes, a retinal assessment should be performed as soon as possible after the first antenatal visit if it has not been done in the preceding 12 months. If any diabetic retinopathy is present, an additional assessment should be performed at 16-20 weeks of gestation. If the first assessment is normal, an assessment should be repeated at 28 weeks of gestation (pg 140).

Grade B, Level $2^{++}$

More frequent assessment may be required in women with poor glycaemic control, hypertension and/or pre-existing retinopathy (pg 140).

GPP

D Women with insulin-treated pregestational diabetes or gestational diabetes mellitus who are receiving corticosteroids for foetal lung maturation should receive additional insulin treatment and close monitoring of glucose levels (pg 140).

Grade D, Level 4 
B Betamimetic drugs (e.g., salbutamol) should not be used for tocolysis in women with diabetes as they may lead to significant hyperglycaemia (pg 140).

Grade B, Level $2^{++}$

D Delivery should be at term for women with pregestational diabetes and gestational diabetes mellitus unless specific obstetric or medical factors dictate otherwise (e.g., foetal macrosomia, poor glycaemic control, polyhydramnios, pre-eclampsia, intrauterine growth restriction) (pg 140).

Grade D, Level 4

D Vaginal delivery is preferable unless there is an obstetric or medical contraindication. The presence of diabetes should not itself constitute an indication for elective caesarean delivery (pg 141).

Grade D, Level 4

D During labour and birth, capillary blood glucose should be monitored every 1-4 hours in women with pregestational diabetes and gestational diabetes mellitus and maintained at $4-7 \mathrm{mmol} / \mathrm{l}$ (pg 141).

Grade D, Level 4

D Intravenous dextrose and insulin infusion is recommended during labour and birth for women whose blood glucose is not maintained at $4-7 \mathrm{mmol} / \mathrm{l}(\mathrm{pg} 141)$.

Grade D, Level 4

D Women with pregestational type 1 diabetes should be considered for intravenous dextrose and insulin infusion from the onset of established labour (pg 141).

Grade D, Level 4

D The neonatologist should be informed of deliveries of infants of women with diabetes so that possible complications like neonatal hypoglycaemia may be monitored and treated early (pg 141).

Grade D, Level 4

C Screening for abnormalities should also be performed in infants of woman with diabetes soon after birth (pg 141).

Grade C, Level $2^{+}$

D Babies of women with diabetes should be fed as soon as possible after birth (within 30 minutes)(pg 141).

Grade D, Level 4

B Infants of women with diabetes who present with clinical signs of hypoglycaemia should have their blood glucose tested and be treated with intravenous dextrose as soon as possible. Close monitoring of blood glucose levels is necessary within the first 48 hours of the baby's life (pg 141).

B Close monitoring of blood glucose levels is necessary within the first 48 hours of the baby's life. Infants of diabetic mothers should be fed early (pg 142).

Grade B, Level 2+

D Women with gestational diabetes should discontinue glucose-lowering treatment immediately after birth and monitor their blood glucose levels (pg 142).

Grade D, Level 4

D Women with insulin-treated pregestational diabetes should reduce their insulin doses immediately after birth and monitor their blood glucose levels carefully to establish the appropriate dose (pg 142).

Grade D, Level 4

GPP

Women who are treated with insulin post-delivery should be informed that they are at increased risk of hypoglycaemia in the postnatal period, especially when breastfeeding, and should be advised to have a meal or snack available before or during feeds (pg 142).

GPP

D Breastfeeding is recommended for infants of women with diabetes (pg 142).

Grade D, Level 3

\section{GPP}

Insulin is recommended for glycaemic control in women with diabetes who breastfeed (pg 142).

GPP

Women with pregestational diabetes who are breastfeeding should continue to avoid any drugs for the treatment of diabetes complications that were discontinued for safety reasons in the pre-conception period (pg 143).

GPP

B All subsequent pregnancies in women with gestational diabetes carry a risk for gestational diabetes mellitus. Early evaluation of glucose tolerance in future pregnancies should be stressed (pg 143).

Grade B, Level 2++

Women with a history of gestational diabetes mellitus should be offered lifestyle advice aimed at diet modification, weight control and increasing physical activity to reduce their risk of subsequent development of diabetes (pg 143).

Grade D, Level 4

For women with gestational diabetes, a 75 g 2-h oral glucose tolerance test (OGTT) should be performed 6-12 weeks postpartum and the woman reclassified and counselled according to criteria accepted in the nonpregnant state (pg 143). 
D Women with a history of gestational diabetes should have lifelong screening for the development of prediabetes or diabetes at least once every 3 years (pg 144).

Grade D, Level 4

D In women with prediabetes or overt diabetes, glucose and lipid profiles should be monitored if low-dose oestrogenprogestin oral contraceptives are used. These should, however, be avoided in women with complications of diabetes and/or other risk factors for vascular disease. Progestin-only preparations may be suitable for these women (pg 144).

Grade D, Level 3

D Low-dose oestrogen-progestin oral contraceptives and intrauterine devices are not contraindicated in women with previous gestational diabetes (pg 144).

Grade D, Level 3

D Oestrogen-progestogen contraceptives should be avoided in women with complications of diabetes and/or other risk factors for vascular disease (pg 144).

Grade D, Level 3

\section{Management of the child and adolescent with diabetes mellitus}

GPP Children and adolescents with suspected diabetes should be referred to a specialist for early assessment, where possible, on the same day (pg 145).

B Children and adolescents with either type 1 diabetes or type 2 diabetes should be provided ongoing and structured diabetes care by a multi-disciplinary diabetes care team (pg 146).

Grade B, Level 2++

Diabetes education should involve the family and child to include learning about blood glucose monitoring, insulin administration, hypoglycaemia and sick day management. As the child matures, diabetes education should emphasise self-care responsibilities shifting from the parent to child, under parental guidance and supervision (pg 146).

GPP

D Include psycho-educational intervention strategies and planned transition in the management of adolescents with diabetes (pg 147)

Grade D, Level 4

GPP Blood glucose targets should be individually determined with a goal to achieving a value as close to normal as possible as there is little age-related scientific evidence for strict glucose targets (pg 148).

GPP

or type 2 diabetes mellitus, and their families should be informed that the target for long term blood glucose control is a $\mathrm{HbA}_{1 \mathrm{c}}$ level of less than $7.5 \%$ or $58.5 \mathrm{mmol} / \mathrm{mol}$ without frequent hypoglycaemia (pg 148).

Grade A, Level 1+

A Children and adolescents with type 1 diabetes should be encouraged to use blood glucose measurements for monitoring of glycaemic control because it is associated with reduced levels of $\mathrm{HbA}_{1 \mathrm{c}}$ (pg 148).

Grade A, Level 1+

GPP Consider the possibility of antecedent nocturnal hypoglycaemia if fasting blood glucose is $<4 \mathrm{mmol} / \mathrm{l}$ (pg 148).

GPP

C

Screening for type 2 diabetes in asymptomatic children and adolescents is not recommended as a public health strategy (pg 149).

Grade C, Level $2^{+}$

\section{GPP}

Type 1 diabetes mellitus in children and adolescents should be managed by an endocrinologist or physician with a special interest in childhood diabetes (pg 149).

GPP

Self-monitoring of blood glucose is an essential tool in the optimal management of childhood and adolescent type 1 diabetes mellitus and should be used in conjunction with insulin treatment (pg 150).

Grade C, Level $2^{+}$

Children and adolescents with type 1 diabetes mellitus should be offered screening for:

- Thyroid disease at diagnosis and annually thereafter.

- Retinopathy annually from the age of 12 years.

- Microalbuminuria annually from the age of 12 years (pg 150).

Grade C, Level 2+

C Children with type 2 diabetes mellitus may initially be treated with lifestyle modification (diet and exercise), unless they are symptomatic or severely hyperglycaemic (pg 151).

\section{Grade C, Level 2+}

C Lifestyle changes in diet and exercise should be recommended for all children with type 2 diabetes mellitus and continued, even after addition of pharmacologic therapy (pg 152).

\section{Grade C, Level $2^{+}$}

C Metformin may be started as the first-line oral agent in children with type 2 diabetes mellitus if blood glucose targets are not achieved. Insulin therapy should be started if oral agents fail to attain target control (pg 152). 
A Metformin is contraindicated in children with impaired renal function and hepatic disease, and should be discontinued with any acute illness associated with dehydration or hypoxaemia (pg 152).

Grade A, Level 1+

A If monotherapy with metformin over 3-6 months has failed, insulin should be added to the treatment (pg 152).

Grade A, Level 1+

C Children and adolescents with type 2 diabetes mellitus should be offered co-morbidity screening for:

- Albuminuria at diagnosis and annually thereafter.

- Hypertension at diagnosis and annually thereafter.

- Dyslipidaemia soon after diagnosis and annually thereafter (pg 153).

Grade C, Level $2^{+}$

GPP Albuminuria should be evaluated at diagnosis and blood pressure should be evaluated at every visit. Confirmed hypertension (BP $>95 \%$ for age, gender and height) or albuminuria can be treated with an angiotensin-converting enzyme (ACE) inhibitor (pg 153).

GPP

GPP Pharmacotherapy is warranted if low density lipoprotein remains elevated $(\geq 3.4 \mathrm{mmol} / \mathrm{l})$ after 6 months of optimised glucose control and diet. Statin therapy has been shown to be safe and effective in children as in adults and should be the first pharmacologic intervention, although long term safety data are not available (pg 153).

GPP

GPP Evaluation for non-alcoholic fatty liver disease (NAFLD) and inquiries about puberty, menstrual irregularities and obstructive sleep apnea should be done at diagnosis and annually thereafter (pg 153).

\section{Diagnosis and management of the adult with type 1 diabetes mellitus}

GPP Patients who are suspected to have type 1 diabetes should be referred to the specialist promptly for assessment (pg 154).

Individuals with type 1 diabetes should have access to a multi-disciplinary team consisting of an endocrinologist, a nurse educator, a dietitian and a mental health professional qualified to provide up to date education and support (pg 155).

A Most people with type 1 diabetes should be treated with multiple dose insulin (MDI) injections (at least three injections per day of prandial insulin and at least one injection per day of basal insulin) or continuous subcutaneous insulin infusion (CSII) (pg 155).

Grade A, Level $1^{+}$

A Most people with type 1 diabetes should use insulin analogues to reduce the risk of hypoglycaemia (pg 155).

Grade A, Level $1^{+}$

A Individuals using rapid-acting insulin by injection or insulin pump should adjust the meal and snack insulin doses based on the carbohydrate content of the meals and snacks (pg 155).

Grade A, Level $1^{+}$

B People with diabetes should receive diabetes selfmanagement education and ongoing support (pg 156).

Grade B, Level $2^{+}$

C Screening of psychosocial functioning, especially anxiety and depression should be performed.Those with positive screening should be referred promptly for treatment (pg 157).

Grade C, Level $2^{+}$

Patients with type 1 diabetes should have thyroid function checked every 1-2 years (pg 157).

GPP

\section{Prevention of type 2 diabetes mellitus}

B Screening for asymptomatic individuals for type 2 diabetes mellitus should be carried out on an opportunistic basis. Testing should be considered in adults of any age who have one or more risk factors for diabetes. In those without risk factors, testing should begin at 40 years (pg 159).

Grade B, Level 2++

A Lifestyle changes with modest weight loss (5-10\% of body weight) and moderate intensity physical activity ( 30 minutes daily) is the treatment of choice with individuals with impaired fasting glucose/impaired glucose tolerance (pg 161).

Grade A, Level $1^{++}$

B Metformin may be considered for the very high risk individual (please refer to chapter on Diagnosis and screening) with impaired fasting glucose/impaired glucose tolerance of age $<60$ and $\mathrm{BMI} \geq 35 \mathrm{~kg} / \mathrm{m}^{2}$ (pg 162).

Grade B, Level $2^{++}$

\section{Clinical quality improvement}

A Measures of process of diabetes care should include the initial and ongoing performance of medical indicators which have been proven to influence long-term outcome (pg 166).

Grade A, Level 1+

GPP Data to measure the outcomes of diabetes management should be obtained from the individual with diabetes (pg 166). 


\section{SINGAPORE MEDICAL COUNCIL CATEGORY 3B CME PROGRAMME} (Code SMJ 201406B)

These questions are based on the full text of the guidelines which may be found at http://www.moh.gov.sg/content/moh_web/ healthprofessionalsportal/doctors/guidelines/cpg_medical.html

Question 1. With regards to diagnosis of diabetes mellitus:

(a) In patients with hyperglycaemic crisis, diabetes mellitus can be diagnosed without further testing.

(b) Diabetes mellitus can be diagnosed if fasting plasma glucose is $6.5 \mathrm{mmol} / \mathrm{l}$.

(c) When two different tests are available for the same patient and the results for both tests are above the diagnostic thresholds, the diagnosis of diabetes is confirmed.

(d) Fasting plasma glucose measured in an accredited laboratory is the preferred test for the diagnosis of diabetes mellitus.

Question 2. With regards to lifestyle modification:

(a) Medical nutrition therapy in diabetes only addresses glycaemic control.

(b) A diet for diabetes should contain a good balance of carbohydrate, protein and fat.

(c) If weight reduction is needed, it should be attempted rapidly (1.00 to $2.0 \mathrm{~kg} /$ week).

(d) All individuals with type 2 diabetes should undertake at least 100 mins/week of moderate to vigorous aerobic exercise.

Question 3. With regards to pharmacotherapy:

(a) All patients with type 1 diabetes need insulin treatment.

(b) Biguanides (metformin) decrease hepatic glucose release, enhance peripheral glucose disposal and delay glucose absorption.

(c) In patients type 2 diabetes, one does not need to monitor for adverse events such as hypoglycaemia or fluid retention.

(d) Metformin is the preferred choice for first-line oral glucose lowering therapy.

Question 4. When assessing glycaemic control:

(a) Self-monitoring of blood glucose should be considered for pregnant patients with pre-existing diabetes.

(b) Self-monitoring of blood glucose should be carried out 3 or more times daily for patients with type 1 diabetes.

(c) Self-monitoring of urine glucose is recommended for monitoring of glycaemic status.

(d) Glycated haemoglobin ( $\mathrm{HbA} 1 \mathrm{c})$ should be performed routinely in all patients with diabetes, at initial assessment and then as part of follow-up care.

Question 5. In terms of reducing the risk of additional complications in patients with diabetes:

(a) To reduce the risk or slow the progression of nephropathy, optimised blood pressure control is recommended.

(b) The recommended time of first eye examination is within one year after diagnosis of diabetes once patient is aged ten years or older.

(c) All individuals with diabetes should receive an annual foot examination to identify high-risk foot conditions.

(d) Women with diabetes who are in the reproductive age group should receive prepregnancy counselling.

\section{Doctor's particulars:}

Name in full

MCR number

Specialty:

Email address

\section{SUBMISSION INSTRUCTIONS:}

(1) Log on at the SMJ website: http://www.sma.org.sg/publications/smjcurrentissue.aspx and select the appropriate set of questions. (2) Provide your name, email address and MCR number. (3) Select your answers and click "Submit".

RESULTS:

(1) Answers will be published in the SMJ August 2014 issue. (2) The MCR numbers of successful candidates will be posted online at the SMJ website by 4 August 2014. (3) Passing mark is $60 \%$. No mark will be deducted for incorrect answers. (4) The SMJ editorial office will submit the list of successful candidates to the Singapore Medical Council. (5) One CME point is awarded for successful candidates.

Deadline for submission: (June 2014 SMJ 3B CME programme): 12 noon, 28 July 2014. 